\title{
Cervical Spinal Canal Stenosis and Central Disc Herniation C3/4 in a Man with Primary Complaint of Thigh Pain
}

\author{
$\begin{array}{lll}\text { R. Akhavan-Sigari } & \text { V. } \text { Rohde }^{1} \quad \text { A. Alaid }\end{array}$ \\ ${ }^{1}$ Department of Neurosurgery, University Medical Center Göttingen, \\ Georg-August-University Göttingen, Göttingen, Germany \\ J Neurol Surg Rep 2013;74:101-104.
}

\author{
Address for correspondence Reza Akhavan-Sigari, BC Neurosurgeon, \\ Attending Neurosurgeon. Department of Neurosurgery, University \\ Medical Center Göttingen, Georg-August-University Göttingen, \\ Robert-Koch-Strasse 40, 37075 Göttingen, Germany \\ (e-mail: reza.akhavan-sigari@med.uni-goettingen.de).
}

\begin{abstract}
Keywords

- cervical disc herniation

- thigh pain

Purpose The purpose of this report is to describe the clinical course of a patient who had a primary complaint of isolated right lateral thigh pain 3 years prior that was nonresponsive to conservative measures. Hypoesthesia in the lateral side of the right forearm, deltoid and biceps, as well as triceps paresis, was also diagnosed.

Results Immediately after surgery, the patient reported substantial improvement in his right thigh symptoms. The signs and symptoms associated with the right C6 and C7 radiculopathy did improve.

Conclusion Although the presentation described in this case is somewhat unique, the eventual myelopathic signs and symptoms were not. It was these myelopathic signs and symptoms that led to cervical magnetic resonance imaging, the diagnosis of cervical cord compressive myelopathy, and surgical management.
\end{abstract}

\section{Introduction}

We report on a patient with multisegmental spinal stenosis with maximal narrowing at C3-4 via multiple disk herniation presenting as the chief complaint of isolated right ventral thigh pain; hypoesthesia in the lateral side of the right forearm, deltoid, and biceps; and triceps paresis who was managed with ventral discectomy C3-4 and fusion and significantly improved postoperatively.

Cervical cord compressive myelopathy is characterized by spinal cord compression due to narrowing of the sagittal diameter of the spinal canal secondary to congenital and (mostly) degenerative changes in the cervical spine. ${ }^{1}$ Cervical cord compressive myelopathy is the most common cause of spinal cord dysfunction in older individuals. ${ }^{2}$ Cervical disc herniation is characterized by a bulge or rupture of the disc material into the spinal canal, which causes abnormal pressure on the nerve at that level, leading to symptoms mainly in the neck and one arm or hand. Diagnosing patients with cervical cord compressive myelopathy in a timely manner can be challenging due to varying clinical presentations, the absence of pathognomonic findings, and symptoms that are usually insidious in nature characterized by disability levels that may not significantly change for long periods. ${ }^{3}$ Additionally, diagnosis can be further complicated because several other conditions can present in a similar manner as cervical cord compressive myelopathy. ${ }^{2}$ The purpose of this report is to describe the clinical course of a patient who had a primary complaint of isolated right ventral thigh pain 3 years prior that was nonresponsive to conservative measures. Hypoesthesia in the lateral side of the right forearm, deltoid, and biceps, as well as triceps paresis, was diagnosed. The patient was subsequently diagnosed with cervical cord compressive myelopathy. After surgical management, the patient's right ventral thigh pain ceased.

\section{Case Report}

A 59-year-old man presented with a primary complaint of right lateral thigh pain, which was limiting his ability to perform activities of daily living. The thigh symptoms, which received

December 21, 2012

accepted

May 20, 2013

published online

July 12, 2013
DOI http://dx.doi.org/

10.1055/s-0033-1349202. ISSN 2193-6358. (c) 2013 Georg Thieme Verlag KG
Stuttgart · New York

License terms

(1) $\Theta \circledast$ 
he described as intermittent, diffuse pain with feelings of cramping and swelling, had been present for more than 3 years.

The patient also reported left deltoid atrophy and intermittent numbness and tingling in the right forearm and the whole right hand.

There was no history of trauma or metabolic disorders. On examination, he had an atrophy of the right deltoid muscle with a manual muscle tent (MMT) grade 2, right biceps weakness with a MMT grade 3, and right triceps weakness with a MMT grade 4. Light touch was impaired from C4 to C6 dermatomes on the right side and on the right lateral thigh region. Pain and temperature were impaired from the $\mathrm{C} 5$ to C7 dermatome. Joint position sense and vibration sense were not impaired over the lower limbs bilaterally. Deep tendon reflexes were exaggerated over the right upper and lower limbs, and left lower limb reflexes were diminished. There was mild tenderness over the cervical spine. The patient had undergone recent neurologic consultations, and conservative management was recommended. He denied weight change, fever/chills/sweats, bowel/bladder changes, or difficulty maintaining balance with walking. Past medical history was unremarkable, and his general health was excellent. He was not taking any medications. The patient demonstrated normal gait. Electromyography for the nerve roots C5, C6, C7 on the right side excluded a denervation of the nerve root.

Somatosensory evoked potential (SSEP) and motor-evoked potential (MEP) studies were performed, and they revealed no changes in the SSEP for the lower extremities. Baseline lower extremity responses were within normal limits with large amplitude values. No change was seen in the latency or amplitude of the SSEP responses, confirming intact somatosensory function.

Magnetic resonance imaging (MRI) of the lumbar spine has shown some degenerative changes and paramedical disc protrusion at level L4/5. No visible nerve involvement (-Figs. 1 and 2). Sensory evoked potentials of the tibial nerve on both sides showed slight prolongation that suggested a possible myelopathy. We could not find any correlation between the right thigh pain and the lumbar MRI findings.

MRI of the cervical spine demonstrated moderately severe spinal stenosis at the $\mathrm{C} 4 / 5, \mathrm{C} 5 / 6$ levels with maximum narrowing at C3/4 (-Figs. 3 and 4). There was no apparent laterality of the cord compression that might account for the unilateral right lower extremity symptoms. The patient was diagnosed with cervical cord compressive myelopathy at C3/4 and underwent a ventral discectomy with cage implantation C3/4. At surgery, the anterior longitudinal ligament was found to be perforated. A medial disc herniation was seen, compressing the dural sac.

Immediately after surgery, the patient reported substantial improvement in his right thigh symptoms. The signs and symptoms associated with the right $\mathrm{C} 6$ and $\mathrm{C} 7$ radiculopathy did improve. At 3 months after surgery, the patient had no complaints of right lateral thigh symptoms and was able to perform all activities of daily living without limitation.

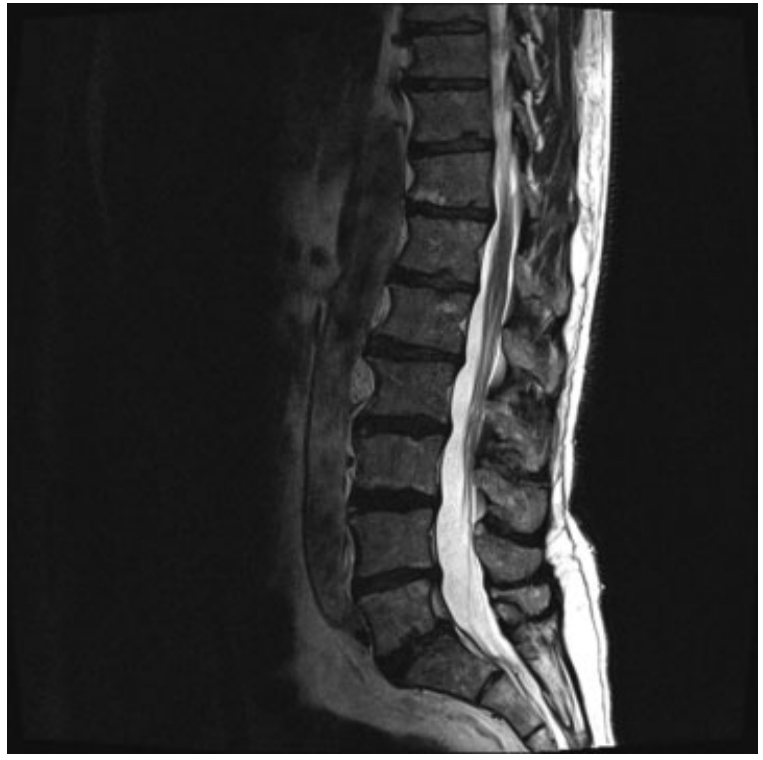

Fig. 1 T2-weighted sagittal magnetic resonance imaging of the lumbar spine shows the normal lumbar curvature. Except for some degenerative changes and paramedical disc protrusion at level L4/5; the vertebral bodies, spinal cord, cerebrospinal fluid, and posterior paraspinal muscles are normal. Slight intraosseous disc hernia was also seen at level L5/S1.

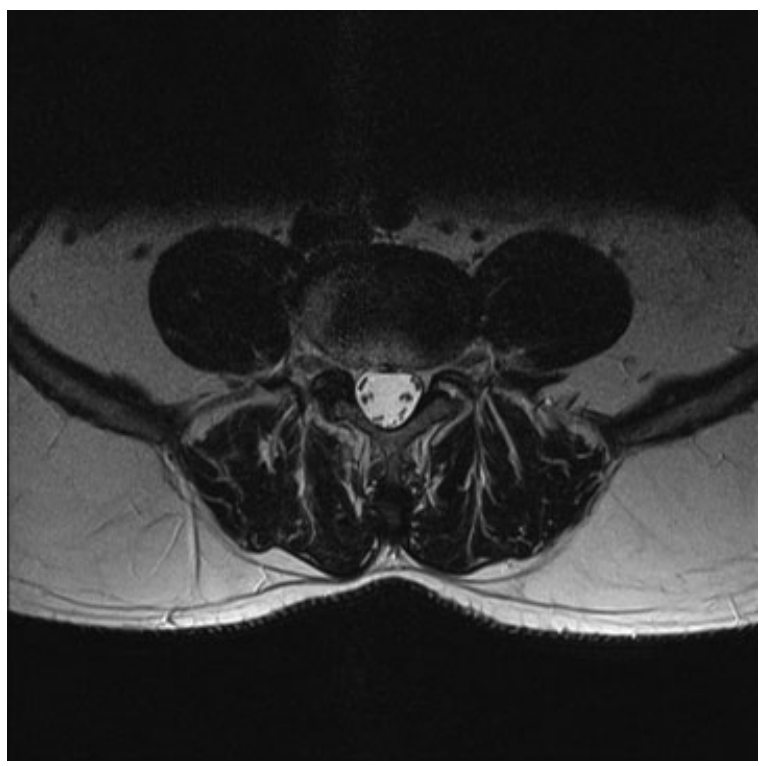

Fig. 2 T2-weighted axial magnetic resonance scan at a lumbar level showing no visible nerve involvement but a slight paramedial disc protrusion at level L4-5.

\section{Discussion}

Failure to accurately identify patients with cervical cord compressive myelopathy in a timely manner can result in progressive symptoms that may not be effectively treated with conservative or surgical interventions. ${ }^{4}$

Surgical treatment has been shown to retard the effects of cervical cord compressive myelopathy when it is managed in a timely fashion. ${ }^{3}$ 


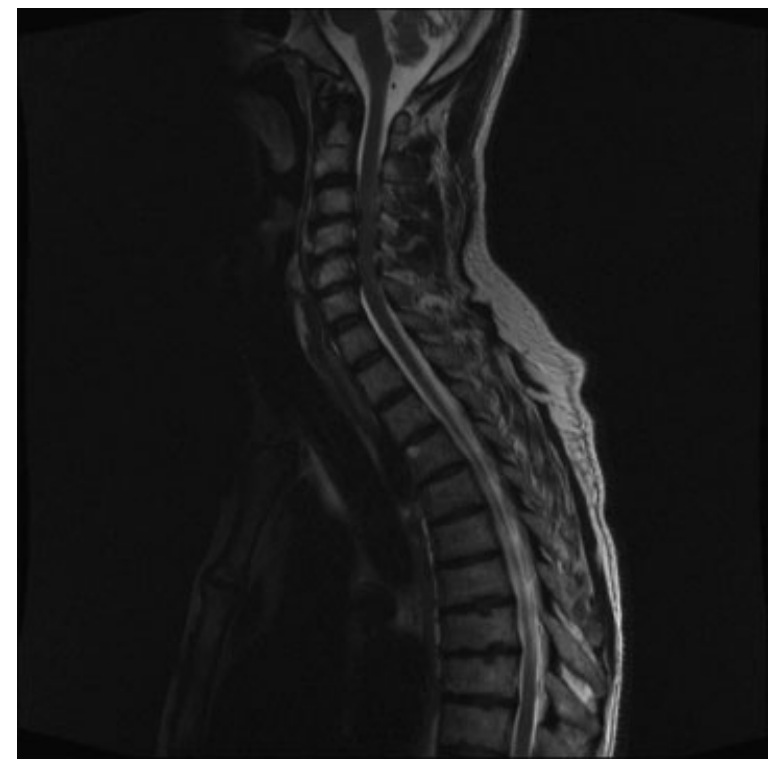

Fig. 3 Sagittal T2-weighted magnetic resonance imaging of the cervical spine, which demonstrated moderately severe spinal stenosis at the C3-C4, C4-C5, and C5-C6 levels, with less severe spinal stenosis at the C5-C6 level. There was also increased signal in the anterior vertebral body of $\mathrm{T} 3$, which were thought to represent small hemangiomas

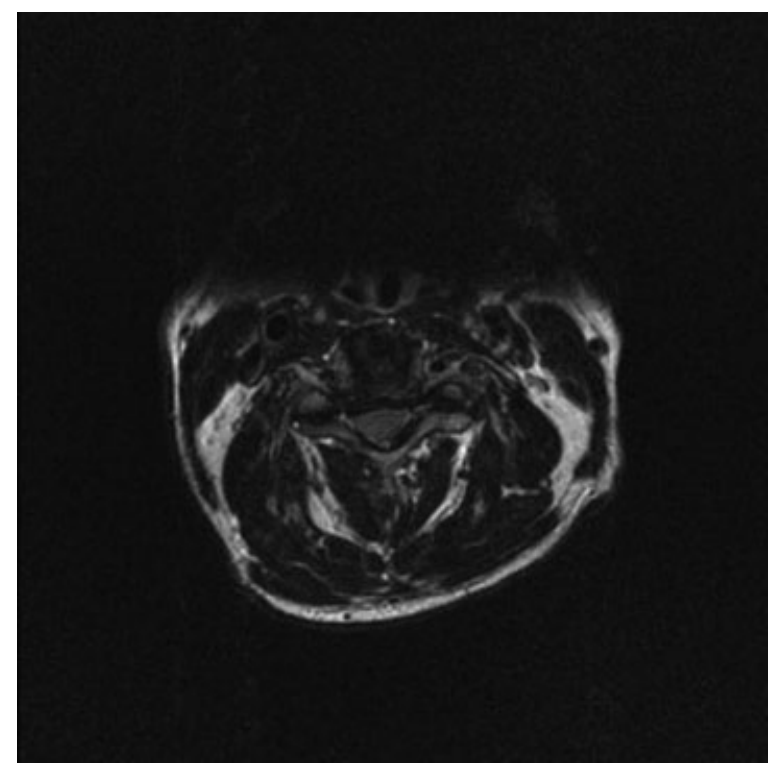

Fig. 4 T2-weighted magnetic resonance axial image at C3-C4 demonstrated central canal stenosis and obliteration of the subarachnoid space.

In this case, the chief complaint was isolated right lateral thigh pain. Right-sided deltoid, biceps, and triceps paresis was also seen, which was thought to be primarily related to transient ischemic attack. Brain MRI revealed no ischemic changes. However, it was not clear whether the right lateral thigh pain and the right upper extremity weakness was related to the transient ischemic attack. The diagnosis of cervical cord compressive myelopathy was not considered until the patient began to report subtle gait disturbances during activities of daily living.
Previous authors have reported that subtle gait disturbances are the most common symptoms associated with cervical cord compressive myelopathy. ${ }^{5}$

Subsequent physical examination findings revealed a positive Romberg test, bilateral upper extremity hyperreflexia right $>$ left, bilateral Babinski reflex, and a multidermatomal decrease in upper extremity sensation during pinprick testing. Therefore, although the presentation was somewhat unique, the patient's myelopathic signs and symptoms were not, and it was these signs and symptoms that led to cervical MRI.

Once the diagnosis of cervical cord compressive myelopathy with maximum narrowing at C3-4 was made, the patient underwent anterior cervical discectomy and fusion C3-4, and his right lateral thigh pain, gait, and the biceps and triceps paresis markedly improved.

It has been reported that diagnosing cervical cord compressive myelopathy is especially difficult in patients with lower extremity pain alone without neurologic symptoms or in patients whose lower extremity symptoms are thought to be related to the lumbar spine ${ }^{6,7}$

In patients with recalcitrant lower extremity pain that is not clearly related to the lumbar spine or musculoskeletal structures of the lower extremity, cervical cord compressive myelopathy should be considered in the differential diagnosis.

Review of the literature revealed only a few reports of lower extremity pain caused by cervical cord compressive myelopathy. ${ }^{6-9}$

Although the precise mechanism is not clearly understood, irritation of the spinothalamic tract has been proposed as a cause. ${ }^{6,7}$ To facilitate the diagnosis of cervical cord compressive myelopathy as a cause for lower extremity symptoms, some authors have attempted to describe the associated clinical symptoms. It has been reported that the pain is usually described as burning, boring, or aching. ${ }^{9}$ The pain is usually bilateral and diffuse, ${ }^{6,8}$ if the pain is unilateral, it is usually contralateral to the side of cord compression. ${ }^{7,8}$ Additionally, in older patients, the pain presentation may not be consistent with other likely musculoskeletal disorders, such as osteoarthritis. ${ }^{9}$

Although these findings may be helpful, a conclusive diagnostic test is unavailable and pain relief after surgery is the only confirmation that the pain was related to cervical cord compressive myelopathy. ${ }^{9}$

This case study should be interpreted with caution because we cannot infer a cause-and-effect relationship between the cervical cord compressive myelopathy and right lateral thigh. Furthermore, it is possible that the outcome described for this patient was coincidental or related to placebo effect. Because there are only a few reports of lower extremity pain being associated with cervical cord compressive myelopathy, ${ }^{10-12}$ a series of patients might serve as better evidence to support a causal relationship between cervical cord compressive myelopathy and lower extremity pain.

\section{Conclusion}

Cervical herniated disc develops usually in the 30-to-50-yearold age group. Although a cervical herniated disc may originate 
from some sort of trauma or injury to the cervical spine, the symptoms, including arm pain, commonly start spontaneously. Appropriate diagnosis and treatment of cervical cord compressive myelopathy may avoid unnecessary diagnostic imaging, medical evaluations, invasive procedures, and potential neurologic complications. Although the presentation described in this case is somewhat unique, the eventual myelopathic signs and symptoms were not. It was these myelopathic signs and symptoms that led to cervical MRI, the diagnosis of cervical cord compressive myelopathy, and surgical management.

\section{References}

1 Gorter K. Influence of laminectomy on the course of cervical myelopathy. Acta Neurochir (Wien) 1976;33(3-4):265-281

2 Gross J, Benzel E. Dorsal surgical approach for cervical spondylotic myelopathy. In: Camins MD, ed. Techniques in Neurosurgery. Philadelphia: Lippincott Williams \& Wilkins; 1999:162-176

3 Kobayashi S. "Tract pain syndrome" associated with chronic cervical disc herniation. Hawaii Med J 1974;33(10):376-381

4 Langfitt TW, Elliott FA. Pain in the back and legs caused by cervical spinal cord compression. JAMA 1967;200(5):382-385
5 McCormick WE, Steinmetz MP, Benzel EC. Cervical spondylotic myelopathy: make the difficult diagnosis, then refer for surgery. Cleve Clin J Med 2003;70(10):899-904

6 Neo M, Ido K, Sakamoto T, Matsushita M, Nakamura T. Cervical disc herniation causing localized ipsilateral popliteal pain. J Orthop Sci 2002;7(1):147-150

7 Scott M. Lower extremity pain simulating sciatica; tumors of the high thoracic and cervical cord as causes. J Am Med Assoc 1956;160(7):528-534

8 Yoshimatsu H, Nagata K, Goto H, et al. Conservative treatment for cervical spondylotic myelopathy. prediction of treatment effects by multivariate analysis. Spine J 2001;1(4):269-273

9 Young WF. Cervical spondylotic myelopathy: a common cause of spinal cord dysfunction in older persons. Am Fam Physician 2000;62(5):1064-1070, 1073

10 Ito T, Homma T, Uchiyama S. Sciatica caused by cervical and thoracic spinal cord compression. Spine 1999;24(12): 1265-1267

11 Ross MD, Elliott R. Cervical cord compressive myelopathy in a man with a primary complaint of knee pain. J Spinal Cord Med 2010; 33(4):431-434

12 Yeung JT, Johnson JI, Karim AS. [2012] Cervical disc herniation presenting with neck pain and contralateral symptoms: a case report. J Med Case Rep. 2012;6(1):166 